\title{
Degrees of Freedom for the MIMO Interference Channel
}

\author{
Syed A. Jafar \\ Electrical Engineering and Computer Science \\ University of California Irvine, California, 92697-2625 \\ Email: syed@ece.uci.edu
}

\author{
Maralle J. Fakhereddin \\ Department of Electrical Engineering \\ California Institute of Technology, Pasadena, CA 91125 \\ Email: maralle@systems.caltech.edu
}

\begin{abstract}
We explore the available degrees of freedom (DoF) for the two user MIMO interference channel, and find a general inner bound and a genie aided outer bound that give us the exact \# of DoF in many cases. We also study a share-and-transmit scheme and show how the gains of transmitter cooperation are entirely offset by the cost of enabling that cooperation so that the available DoF are not increased.
\end{abstract}

\section{INTRODUCTION}

Multiple input multiple output (MIMO) systems have assumed great importance in recent times because of their remarkably higher capacity compared to single input single output systems. It is well known [1]-[3] that capacity of a point to point (PTP) MIMO system with $M$ inputs and $N$ outputs increases linearly as $\min (M, N)$ at high $\mathrm{SNR}$. For power and bandwidth limited wireless systems, this opens up another dimension - "space" that can be exploited in a similar way as time and frequency. Similar to time division and frequency division multiplexing, MIMO systems present the possibility of multiplexing signals in space. For example, using singular value decomposition (SVD) of a MIMO channel, one can generate parallel channels in space similar to those created by dividing time or frequency into orthogonal slots.

The availability of spatial DoF depends upon two factors: cooperation within inputs/outputs, and channel knowledge. Previous work has shown that in the absence of channel knowledge, spatial DoF are lost [4], [5]. Multiuser systems, with constrained cooperation between inputs/outputs distributed among multiple users, are especially challenging since, unlike PTP case, joint processing is not possible at inputs/outputs. The available spatial DoF are affected by the inability to jointly process the signals at the distributed inputs and outputs. [6] investigated DoF as a function of distributed and partial side information for multiple access (MAC) and broadcast (BC) channels.

In this paper, we quantify the loss in available DoF under the distributed processing constraints imposed by the two user interference channel. It was recently shown in [7] that cooperation between single antenna transmitters does not provide additional multiplexing gain in an interference channel. In this paper, we explore the benefits of transmitter cooperation when the nodes have multiple antennas. We establish a general innerbound and a genie based outerbound on the \# of DoF for MIMO interference channel. For many cases of practical interest, these bounds are shown to be tight and we have the exact \# of DoF. We also consider a simple cooperative scheme to understand why transmitter cooperation does not increase DoF. Through this simple scheme, we are able to show how the benefits of cooperation are completely offset by the cost of enabling it.

\section{Degrees of Freedom Measure}

In order to isolate the impact of distributed processing from channel uncertainty, we assume that channel state is fixed and perfectly known at all transmitters and receivers. Also, we assume that the channel matrices are sampled from a rich scattering environment. Therefore we can ignore the measure zero event that some channel matrices are rank deficient. It is well known that the capacity of a scalar additive white Gaussian noise (AWGN) channel scales as $\log (S N R)$ at high SNR. On the other hand, for a single user MIMO channel with $M$ inputs and $N$ outputs, the capacity growth rate can be shown to be $\min (M, N) \log (S N R)$ at high SNR. This motivates the natural definition of spatial DoF as:

$$
\eta \triangleq \lim _{\rho \rightarrow \infty} \frac{C_{\Sigma}(\rho)}{\log (\rho)},
$$

where $C_{\Sigma}(\rho)$ is the sum capacity (just capacity in case of PTP channels) at SNR $\rho$. In other words, DoF $\eta$ represent the maximum multiplexing gain [3] of the generalized MIMO system. For PTP case, $(M, N)$ DoF are easily seen to correspond to the parallel channels that can be isolated using SVD, involving joint processing at the $M$ inputs and $N$ outputs, i.e.

$$
\eta(\mathrm{PTP})=\min (M, N)
$$

\section{A. The Multiple Access Channel}

The MAC channel is an example of a MIMO system where cooperation is allowed only between the channel outputs. Let the MAC consist of $N$ outputs controlled by the same receiver and 2 users, each controlling $M_{1}$ and $M_{2}$ inputs for a total of $M=M_{1}+M_{2}$ inputs. For the MAC, the available DoF are the same as with perfect cooperation between all users.

$$
\eta(\mathrm{MAC})=\eta(\mathrm{PTP})=\min \left(M_{1}+M_{2}, N\right) .
$$

While the capacity region of the MIMO MAC is well known and the spatial multiplexing gain has also been explored in previous work, we include the following constructive proof to introduce zero forcing $(\mathrm{ZF})$ notation which will be useful in the derivation of our main result for the interference channel. 
$\mathrm{ZF}$, which is normally a suboptimal strategy, is sufficient in this case (as well as in MIMO BC channel) to utilize all DoF. Converse: The converse is straightforward because, for the same \# of inputs and outputs, $\eta$ (MAC) $\leq \eta(\mathrm{PTP})=$ $\min \left(M_{1}+M_{2}, N\right)$. In other words, the lack of cooperation at the inputs can not increase DoF.

Achievability: The $N \times 1$ received signal $\mathbf{Y}$ at the MAC receiver

$$
\mathbf{Y}=\sum_{k=1}^{2} \mathbf{H}^{(k)} \mathbf{X}^{(k)}+\mathbf{N}=\mathbf{V}_{\mathbf{H}^{\dagger}} \mathbf{V}_{\mathbf{X}}+\mathbf{Z},
$$

where $\mathbf{N}$ is the $N \times 1$ AWGN vector, $\mathbf{H}^{(k)}$ is the $N \times M_{k}$ channel matrix for user $k$, and $\mathbf{X}^{(k)}$ is the $M_{k} \times 1$ transmitted vector for user $k . \mathbf{V}_{\mathbf{H}}=V\left(\mathbf{H}^{(\cdot)^{\dagger}}\right)$ is the $\left(M_{1}+M_{2}\right) \times N$ matrix obtained by vertically stacking the matrices $\mathbf{H}^{(1)^{\dagger}}$ and $\mathbf{H}^{(2)^{\dagger}}$. Similarly, $\mathbf{V}_{\mathbf{X}}=V\left(\mathbf{X}^{(\cdot)}\right)$ is the $\left(M_{1}+M_{2}\right) \times 1$ matrix obtained by vertically stacking $\mathbf{X}^{(1)}$ and $\mathbf{X}^{(2)}$. Transforming the output vector

$$
\mathbf{Y}^{\text {new }}=\left(\mathbf{V}_{\mathbf{H}} \mathbf{V}_{\mathbf{H}}^{\dagger}\right)^{-1} \mathbf{V}_{\mathbf{H}} \mathbf{Y}
$$

(using generalized Moore-Penrose inverse) and ignoring the zero gain channels result in the $\min (M, N)$ parallel channels

$$
\mathbf{Y}^{\text {new }}(i)=\mathbf{V}_{\mathbf{X}}(i)+\mathbf{N}^{\text {new }}(i), \quad 1 \leq i \leq \min (M, N),
$$

where $\mathbf{N}^{\text {new }}(i) \sim \mathcal{N}\left(0, \lambda_{i}\right)$ are Gaussian noise terms and $\lambda_{i}$ is the $i^{\text {th }}$ diagonal term of $\left(\mathbf{V}_{\mathbf{H}} \mathbf{V}_{\mathbf{H}}{ }^{\dagger}\right)^{-1}$. The noise terms may be correlated across different channels but the correlations are inconsequential since each channel is encoded and decoded separately. Dividing power equally among the $\min (M, N)$ channels, we can achieve

$$
\begin{aligned}
\eta(\text { MAC }) \geq & \lim _{\rho \rightarrow \infty} \frac{1}{\log (\rho)} \sum_{i=1}^{\min (M, N)} \log \left(1+\frac{\rho}{\min (M, N)} \frac{1}{\lambda_{i}^{2}}\right) \\
= & \lim _{\rho \rightarrow \infty} \frac{1}{\log (\rho)}[\min (M, N) \log (\rho)+ \\
& \left.\sum_{i=1}^{\min (M, N)} \log \left(\frac{1}{\lambda_{i}^{2} \min (M, N)}\right)\right]=\min (M, N)
\end{aligned}
$$

Note that the channel gains or the exact power allocation does not affect the DoF as long as the SNR on each channel is proportional to $\rho$.

Combining the converse and the achievability, we have established that $\eta(M A C)=\min \left(M_{1}+M_{2}, N\right)$.

\section{B. The Broadcast Channel}

The BC channel is an example of a MIMO system where cooperation is allowed only between the channel inputs. Let the BC consist of $M$ inputs controlled by the same transmitter and 2 users, each controlling $N_{1}$ and $N_{2}$ outputs for a total of $N=N_{1}+N_{2}$ outputs. In a similar fashion as the MAC, it is possible to show that by $\mathrm{ZF}$ at the $\mathrm{BC}$ transmitter, $\min (M, N)$ parallel channels can be created, so that the total DoF are the same as with perfect cooperation between all the users.

$$
\eta(\mathrm{BC})=\eta(\mathrm{MAC})=\eta(\mathrm{PTP})=\min (M, N) .
$$

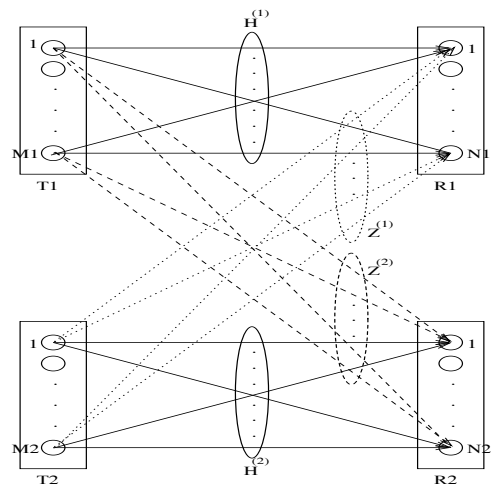

Fig. 1. $\left(M_{1}, N_{1}\right),\left(M_{2}, N_{2}\right)$ Interference channel

\section{INTERFERENCE CHANNEL}

Consider an $\left(M_{1}, N_{1}\right),\left(M_{2}, N_{2}\right)$ interference channel with two transmitters $T_{1}$ and $T_{2}$, and two receivers $R_{1}$ and $R_{2}$, where $T_{1}$ has a message for $R_{1}$ only and $T_{2}$ has a message for $R_{2}$ only. $T_{1}$ and $T_{2}$ have $M_{1}$ and $M_{2}$ antennas respectively. $R_{1}$ and $R_{2}$ have $N_{1}$ and $N_{2}$ antennas respectively. We denote the channels for link 1 with $N_{1} \times M_{1}$ channel gain matrix $\mathrm{H}^{(1)}$, for link 2 by $N_{2} \times M_{2}$ matrix $\mathrm{H}^{(2)}$, for the channel between $T_{1}$ and $R_{2}$ by $N_{2} \times M_{1}$ channel matrix $\mathrm{Z}^{(2)}$, and between $T_{2}$ and $R_{1}$ by $N_{1} \times M_{2}$ matrix $\mathrm{Z}^{(1)}$. Figure 1 shows an illustration of this interference channel. We assume that we arrange the links so that link 1 always has the most \# of antennas either at its transmitter or receiver, i.e. $\max \left(M_{1}, N_{1}\right) \geq \max \left(M_{2}, N_{2}\right)$.

\section{A. Innerbound on the Available Degrees of Freedom}

For the $\left(M_{1}, N_{1}\right),\left(M_{2}, N_{2}\right)$ interference channel we prove the following innerbound on the available DoF.

$$
\begin{aligned}
\eta(\mathrm{INT}) & \geq \min \left(M_{1}, N_{1}\right) \\
& +\min \left(M_{2}-N_{1}, N_{2}\right)^{+} 1\left(M_{1}>N_{1}\right) \\
& +\min \left(M_{2}, N_{2}-M_{1}\right)^{+} 1\left(M_{1}<N_{1}\right),
\end{aligned}
$$

where $1($.$) is the indicator function and (x)^{+}=\max (0, x)$. While we conjecture that this bound is tight for any $M_{1}, N_{1}, M_{2}, N_{2}$, we can prove a converse only with some additional assumptions on the \# of antennas. A general achievability proof is outlined next.

1) Sketch of Achievability Proof: According to our model, either $M_{1} \geq N_{1}, M_{2}, N_{2}$ or $N_{1} \geq M_{1}, M_{2}, N_{2}$. First, we consider the case when $M_{1} \geq N_{1}, M_{2}, N_{2}$.

Step 1: From SVD, $Z^{(2)}=U \Lambda V^{H}$, where $\mathrm{U}$ and $\mathrm{V}$ are $N_{2} \mathrm{x} N_{2}$ and $M_{1} \mathrm{x} M_{1}$ unitary matrices respectively and $\Lambda$ is the diagonal matrix of singular values of $Z^{(2)}$. By applying SVD to $Z^{(2)}$, we decompose the channel into $\min \left(M_{1}, N_{2}\right)$ parallel channels. Therefore, there are $M_{1}-N_{2}$ effective inputs at $T_{1}$ that are not connected to $R_{2}$, and do not cause any interference to $R_{2}$.

Step 2: Similarly, applying SVD to $Z^{(1)}$ creates $\min \left(M_{2}, N_{1}\right)$ parallel connections. There are $\left(M_{2}-N_{1}\right)^{+}$effective inputs at $T_{2}$ that are not connected to $R_{1}$, and therefore do not cause any interference with $R_{1}$. 


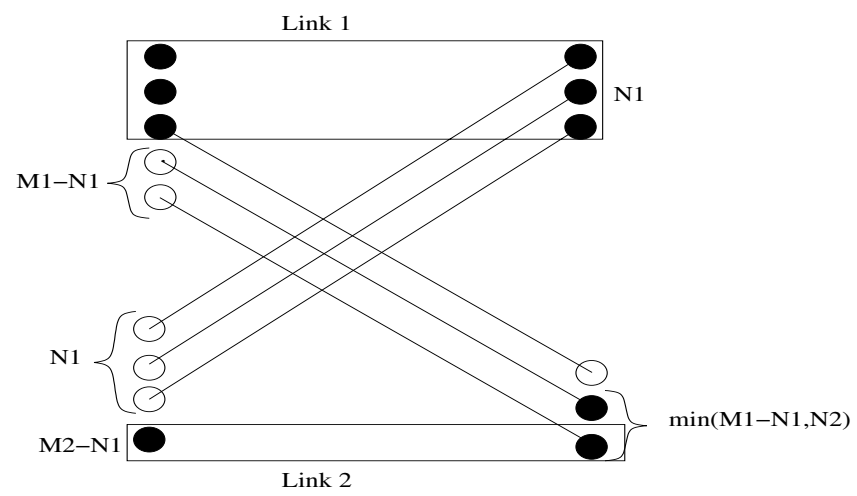

Fig. 2. Achievability proof for $\left(M_{1}, N_{1}\right),\left(M_{2}, N_{2}\right)$ Interference channel when $M_{1} \geq M_{2}, N_{1}, N_{2}$

Step 3: For link 1, all $N_{1}$ effective outputs are used by $R_{1}$. Step 4: $T_{1}$ transmits to $R_{1}$ using $N_{1}$ effective inputs such that at most $\left(N_{1}+N_{2}-M_{1}\right)^{+}$effective inputs that are active are also connected to $R_{2}$.

Step 5: Link 2 uses only those effective inputs/outputs that are not connected to an active effective input/output of link 1.

Step 6: Link 1 is left with $N_{1}$ effective inputs and $N_{1}$ effective outputs, i.e. the \# of DoF for link $1=N_{1}$.

Step 7: For link 2, $T_{2}$ is left with $\left(M_{2}-N_{1}\right)^{+}$effective inputs while $R_{2}$ is left with $\min \left(M_{1}-N_{1}, N_{2}\right)$ effective outputs, i.e. the \# of DoF for link $2=\min \left(M_{2}-N_{1}, \min \left(M_{1}-\right.\right.$ $\left.\left.N_{1}, N_{2}\right)\right)^{+}=\min \left(M_{2}-N_{1}, N_{2}\right)^{+}$since $M_{1} \geq M_{2}$ by assumption. Hence proved.

For the case when $N_{1} \geq M_{1}, M_{2}, N_{2}$, the same logic is followed. Then, the total \# of DoF is $\min \left(M_{1}, N_{1}\right)+$ $\min \left(M_{2}, N_{2}-M_{1}\right)^{+}$. By adding the results from the two cases, we obtain a general achievable proof of (8). An illustration of this proof is shown in figure 2.

\section{B. Outerbounds on the Available Degrees of Freedom}

To start with, notice that a trivial outerbound is obtained from the PTP case, i.e. $\eta($ INT $) \leq \min \left(M_{1}+M_{2}, N_{1}+N_{2}\right)$. Indeed this outerbound coincides with the innerbound when either $\min \left(M_{1}, M_{2}\right) \geq N_{1}+N_{2}$ or $\min \left(N_{1}, N_{2}\right) \geq M_{1}+$ $M_{2}$. In general, while the capacity region of the interference channel is not known even with single antennas at all nodes, various outerbounds have been obtained [8]-[10] that have been useful in finding the capacity region in some special cases [11], [12]. Most of the existing outerbounds are for single antenna systems.

For our purpose, we develop a genie based outerbound for MIMO interference channel where the \# of antennas at either receiver is $\geq$ the \# of transmit antennas at the interfering transmitter, i.e. either $N_{1} \geq M_{2}$ or $N_{2} \geq M_{1}$. We find that, in many cases, this outerbound is sufficiently tight to establish the \# of DoF. Note that for this section, since we do not use the assumption that $\max \left(M_{1}, N_{1}\right) \geq \max \left(M_{2}, N_{2}\right)$, the proof for the cases $N_{1} \geq M_{2}$ or $N_{2} \geq M_{1}$ is identical.

Theorem 1: For the $\left(M_{1}, N_{1}\right),\left(M_{2}, N_{2}\right)$ interference channel with $N_{1} \geq M_{2}$, the sum capacity is bounded above by that of the corresponding $\left(M_{1}, M_{2}, N_{1}\right)$ MAC channel with additive noise $\mathbf{N}^{(\mathbf{1})} \sim \mathcal{N}\left(\mathbf{0}, \mathbf{I}_{\mathbf{N}}\right)$ modified to $\mathbf{N}^{(\mathbf{1})^{\prime}} \sim \mathcal{N}\left(\mathbf{0}, \mathbf{K}^{\prime}\right)$ where

$$
\begin{aligned}
& \mathbf{K}^{\prime}=\mathbf{I}_{\mathbf{N}}-\mathbf{Z}^{(\mathbf{1})}\left(\mathbf{Z}^{(\mathbf{1}) \dagger} \mathbf{Z}^{(\mathbf{1})}\right)^{-1} \mathbf{Z}^{(\mathbf{1}) \dagger}+\alpha \mathbf{Z}^{(\mathbf{1})} \mathbf{Z}^{(\mathbf{1})^{\dagger}} \\
& \alpha=\min \left(\frac{1}{\sigma_{\max }^{2}\left(\mathbf{Z}^{(\mathbf{1})}\right)}, \frac{1}{\sigma_{\max }^{2}\left(\mathbf{H}^{(\mathbf{2})}\right)}\right) \\
& \text { Proof: }
\end{aligned}
$$

Let us define

$$
\begin{aligned}
& \mathbf{N}_{\mathbf{a}}^{(\mathbf{1})} \sim \mathcal{N}\left(\mathbf{0}, \mathbf{I}_{\mathbf{N}}-\mathbf{Z}^{(\mathbf{1})}\left(\mathbf{Z}^{(\mathbf{1}) \dagger} \mathbf{Z}^{(\mathbf{1})}\right)^{-1} \mathbf{Z}^{(\mathbf{1}) \dagger}\right) \\
& \mathbf{N}_{\mathbf{b}}^{(\mathbf{1})} \sim \mathcal{N}\left(\mathbf{0}, \mathbf{Z}^{(\mathbf{1})}\left(\mathbf{Z}^{(\mathbf{1}) \dagger} \mathbf{Z}^{(\mathbf{1})}\right)^{-1} \mathbf{Z}^{(\mathbf{1}) \dagger}-\alpha \mathbf{Z}^{(\mathbf{1})} \mathbf{Z}^{(\mathbf{1})^{\dagger}}\right) \\
& \mathbf{N}_{\mathbf{c}}^{(\mathbf{1})} \sim \mathcal{N}\left(\mathbf{0}, \alpha \mathbf{Z}^{(\mathbf{1})} \mathbf{Z}^{(\mathbf{1})^{\dagger}}\right)
\end{aligned}
$$

as three $N \times 1$ jointly Gaussian and mutually independent random vectors. The positive semidefinite property of the respective covariance matrices is easily established from the definition of $\alpha$.

Without loss of generality we assume

$$
\begin{aligned}
\mathbf{N}^{(1)} & =\mathbf{N}_{\mathbf{a}}^{(\mathbf{1})}+\mathbf{N}_{\mathbf{b}}^{(\mathbf{1})}+\mathbf{N}_{\mathbf{c}}^{(\mathbf{1})} \\
\mathbf{N}^{(1)^{\prime}} & =\mathbf{N}_{\mathbf{a}}^{(\mathbf{1})}+\mathbf{N}_{\mathbf{c}}^{(\mathbf{1})}
\end{aligned}
$$

Furthermore, because $\mathbf{N}^{(\mathbf{1})}$ and $\mathbf{N}^{(\mathbf{2})}$ have the same marginal distributions and the capacity of the interference channel does not depend on the correlation between $\mathbf{N}^{(\mathbf{1})}$ and $\mathbf{N}^{(\mathbf{2})}$, the capacity region is not affected if we assume

$$
\mathbf{N}^{(1)}=\mathbf{N}^{(\mathbf{2})} \text {. }
$$

Since a part of the proof is similar to the corresponding proof for the single antenna case, we will summarize the common steps, and emphasize only the part that is unique to MIMO interference channel. Consider any achievable scheme for any rate point within the capacity region of the interference channel, so that $R_{1}$ and $R_{2}$ can correctly decode their intended messages from their received signals with sufficiently high probability.

Step 1: We replace the original additive noise $\mathbf{N}^{(\mathbf{1})}$ at $R_{1}$ with $\mathbf{N}^{(\mathbf{1})^{\prime}}$ as defined in Theorem 1. We argue that this does not make the capacity region smaller because the original noise statistics can easily be obtained by locally generating and adding noise $\mathbf{N}_{\mathrm{b}}^{(\mathbf{1})}$ at $R_{1}$. Therefore, since $R_{1}$ was originally capable of decoding its intended message with noise $\mathbf{N}^{(\mathbf{1})}$, it is still capable of decoding its intended message with $\mathbf{N}^{(\mathbf{1})^{\prime}}$.

Step 2: Suppose that a genie provides $R_{2}$ with side information containing the entire codeword $\mathbf{X}^{(1)}$. Since $\mathbf{X}^{(2)}$ is independent of $\mathbf{X}^{(1)}, R_{2}$ simply subtracts out the interference from its received signal. Thus, the channel $\mathbf{Z}^{(2)}$ can be eliminated without making the capacity region smaller.

Step 3: By our assumption, $R_{1}$ can decode its own message and therefore it can subtract $\mathbf{X}^{(1)}$ from its own received signal as well. In this manner, after the interfering signals have been subtracted out we have

$$
\begin{aligned}
& \mathbf{Y}^{(1)}=\mathbf{Z}^{(\mathbf{1})} \mathbf{X}^{(\mathbf{2})}+\mathbf{N}^{(\mathbf{1})^{\prime}}, \\
& \mathbf{Y}^{(2)}=\mathbf{H}^{(\mathbf{2})} \mathbf{X}^{(\mathbf{2})}+\mathbf{N}^{(\mathbf{2})} .
\end{aligned}
$$


To complete the proof we need to show that if $R_{2}$ can decode $\mathrm{X}^{(2)}$ then so can $R_{1}$. This would imply that $R_{1}$ can decode both messages, hence giving us the MAC outer bound.

Step 4: Without loss of generality, let us perform SVD $\mathbf{H}^{(2)}=$ $\mathbf{U}^{(2)} \Lambda^{(\mathbf{2})} \mathbf{V}^{(\mathbf{2})}$ on the channel between $T_{2}$ and $R_{2}$. This is a lossless operation that leads to:

$$
\mathbf{Y}^{(2) \text { new }}=\mathbf{X}^{(\mathbf{2}) \text { new }}+\left(\boldsymbol{\Lambda}^{(\mathbf{2})}\right)^{-1} \mathbf{N}^{(\mathbf{2})}
$$

where $\mathbf{X}^{(2) \text { new }}=\mathbf{V}^{(2)} \mathbf{X}^{(2)}$.

To save space we allow some notation abuse as we use generalized inverse and ignore the terms that correspond to zero diagonal channel gains in $\boldsymbol{\Lambda}^{(\mathbf{2})}$. Note that these channels are useless for $R_{2}$. Also, we use the same symbol for rotated versions of noise that are statistically equivalent.

Step 5: Next, we show that $R_{1}$ can obtain a stronger channel to $\mathbf{X}^{(2) n e w}$ so that if $R_{2}$ can decode it, so can $R_{1}$. To this end, let $R_{1}$ use $\mathrm{ZF}$ to obtain:

$$
\begin{aligned}
\mathbf{Y}^{(1) \text { new }} & =\mathbf{X}^{(\mathbf{2}) \text { new }}+\mathbf{V}^{(\mathbf{2})}\left(\mathbf{Z}^{(\mathbf{1}) \dagger} \mathbf{Z}^{(\mathbf{1})}\right)^{-1} \mathbf{Z}^{(\mathbf{1}) \dagger} \mathbf{N}^{(\mathbf{1})^{\prime}} \\
& =\mathbf{X}^{(\mathbf{2}) \text { new }}+\alpha \mathbf{N}^{(\mathbf{2})}
\end{aligned}
$$

Now both $R_{1}$ and $R_{2}$ have a diagonal channel with input $\mathbf{X}^{(\mathbf{2}) \text { new }}$ and uncorrelated additive white noise components on each diagonal channel. Moreover, the strongest channel for $R_{2}$ has noise $\frac{1}{\sigma_{\max }^{2}\left(\mathbf{H}^{(2)}\right)}$. However the noise on any channel for $R_{1}$ is only $\alpha$ which is smaller. Thus, we argue once again that $R_{1}$ can locally generate noise and add it to its received signal to create a statistically equivalent noise signal as seen by $R_{2}$. In other words, $R_{1}$ has a less noisy channel to $T_{2}$ and therefore can decode any signal that $R_{2}$ can. Since $R_{1}$ can decode $T_{1}$ 's message by assumption, we have the MAC outerbound.

The MAC outerbound leads directly to the following outerbound on the \# of DoF.

Corollary 1: For the $\left(M_{1}, N_{1}\right),\left(M_{2}, N_{2}\right)$ interference channel with $N_{1} \geq M_{2}$, the \# of DoF $\eta($ INT $) \leq \min \left(M_{1}+\right.$ $\left.M_{2}, N_{1}\right)$. Similarly, if $N_{2} \geq M_{1}$, then $\eta($ INT $) \leq \min \left(M_{1}+\right.$ $\left.M_{2}, N_{2}\right)$.

The outerbound and innerbound are tight in many cases where we have the exact \# of DoF. Some examples are provided in the following table.

\begin{tabular}{|c|c|c|}
\hline$\left(M_{1}, N_{1}\right)$ & $\left(M_{2}, N_{2}\right)$ & $\eta(I N T)$ \\
\hline$(1,1)$ & $(1,1)$ & 1 \\
\hline$(1,2)$ & $(1,2)$ & 2 \\
\hline$(2,1)$ & $(2,1)$ & 2 \\
\hline$(1,2)$ & $(2,1)$ & 1 \\
\hline$(3,2)$ & $(2,3)$ & 2 \\
\hline$(2,3)$ & $(2,3)$ & 3 \\
\hline$(2,3)$ & $(1,3)$ & 3 \\
\hline$(2,2)$ & $(3,2)$ & 2 \\
\hline
\end{tabular}

\section{EFFECT OF TRANSMIT COOPERATION ON THE NUMBER OF DEGREES OF FREEDOM}

Comparing the interference channel and the BC channel obtained by full cooperation between the transmitters, it is clear that the available DoF are severely limited by the lack of transmitter cooperation in the interference channel. As an example, consider the interference channel with $\left(M_{1}, N_{1}\right)=$ $(n, 1)$ and $\left(M_{2}, N_{2}\right)=(1, n)$. From the preceding section we know there is only one available degree of freedom in this channel. However, if full cooperation between the transmitters is possible the resulting $\mathrm{BC}$ channel has $\left(M, N_{1}, N_{2}\right)=$ $(n+1,1, n)$. The \# of DoF is now $n+1$. Therefore, transmitter cooperation would seem highly desirable. Rather surprisingly, it has been shown recently [7] that for the $(1,1),(1,1)$ interference channel, allowing the transmitters to cooperate through a wireless link between them (even with full duplex operation), does not increase DoF. For MIMO interference channels, as suggested by the example above, the potential benefits of cooperation are even stronger and it is not known if transmitter cooperation can increase DoF. The capacity results of [7] do not seem to allow direct extensions to MIMO interference channels.

To gain insights into the cost and benefits of cooperation in a MIMO interference channel, we consider a specific scheme where transmitters first share their information in a full duplex mode as a MIMO channel (step 1) and subsequently transmit together as BC channel. We will refer to this scheme as the share-and-transmit scheme.

\section{A. Degrees of Freedom with Share-and-Transmit}

Consider an $(M, N),(M, N)$ interference channel $(M \leq$ $N)$. Also assume that each transmitter is sending information with rate $R$. Note that while we make the preceding simplifying assumptions for simplicity of exposition, the following analysis and the main result extend directly to the general case of unequal \# of antennas and unequal rates.

From (8), we know that the \# of DoF for this interefernce channel with no transmitter cooperation is $\min (M, N)+$ $\min (M, N-M)^{+}=M+\min (M, N-M)^{+}$. For the shareand-transmit scheme, we compute DoF as follows. We first find the capacity of the sharing link $C_{s}$ and the capacity of transmission $C_{t}$. Then, we find the total capacity of the system $C$ by evaluating the total amount of data transmitted divided by the total time it requires to transmit this data, i.e.

$$
C=\frac{2 R}{\frac{R}{C_{s}}+\frac{2 R}{C_{t}}} .
$$

Dividing by $\log (\mathrm{SNR})$ where $\mathrm{SNR}$ is large, we obtain the total \# of DoF as

$$
\lim _{S N R \rightarrow \infty} \frac{C}{\log S N R}=\frac{2}{\frac{1}{D O F(\text { sharing })}+\frac{2}{D O F(\text { transmit })}} .
$$

The \# of DoF for the sharing link is that of MIMO PTP channel with $M$ transmit and receive antennas $=\min (M, M)=$ $M$. After transmitters share their information, they can fully cooperate as a $(2 M, N, N)$ BC channel. The \# of DoF for this channel is $\min (2 M, 2 N)=2 \min (M, N)$. Therefore (11), which gives the total \# of DoF for the share-and-transmit scheme, becomes $\frac{2 M \min (M, N)}{M+\min (M, N)}=M$. Note that,

$$
M+\min (M, N-M)^{+} \geq M \text {. }
$$




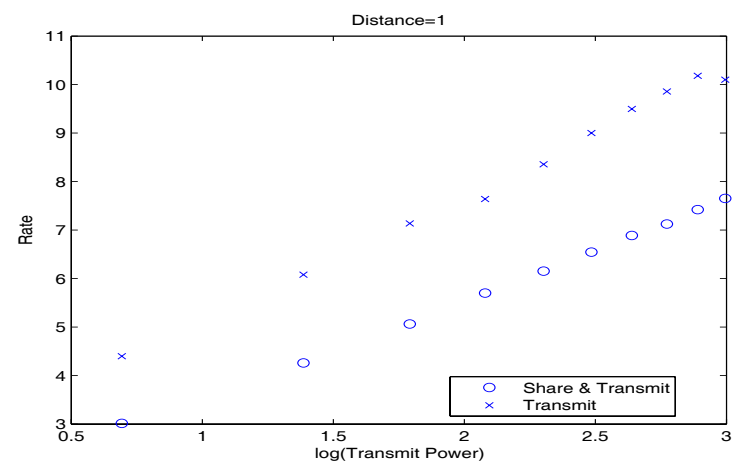

Fig. 3. Rate vs $\log$ (Transmit Power) with same distance.

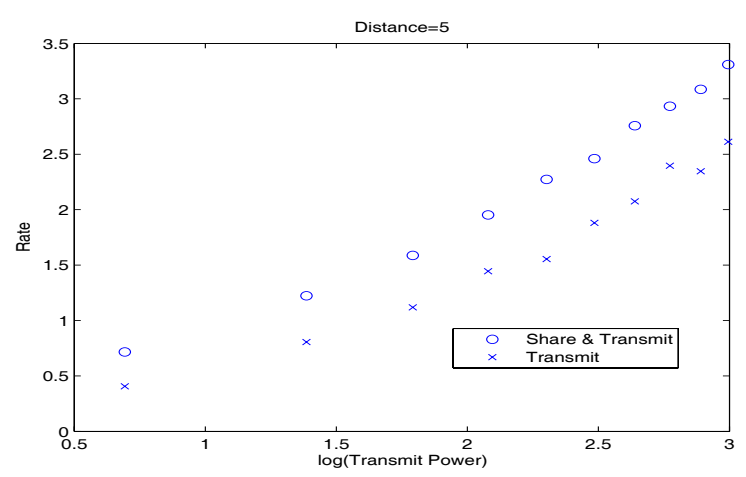

Fig. 4. Rate vs $\log ($ Transmit Power) with $5 \times$ distance for transmitting.

Therefore, we conclude that (for this specific scheme) transmitter cooperation in the high SNR regime does not provide any advantage to the \# of DoF in the MIMO interference channel.

\section{Simulation Results}

In this section, we verify the result discussed in the previous section, and discuss the effect of transmitter cooperation when the sharing links between the transmitters are stronger than the transmission links. For simplicity, we consider a $(4,1),(4,1)$ interfernce channel, and plot the rate versus the logarithm of the transmit power. Note that we assume the noise to be 0mean unit-variance Gaussian additive noise.

The share-and-transmit scheme is implemented as explained in section IV-A. For the no cooperation scheme, $T_{1}$ has a message for $R_{1}$ only and dedicates its available power to its link with $R_{1}$. The same is true for $T_{2}$ and $R_{2}$. Note that since the transmit signal space is much larger than the receive signal space, $T_{1}$ can decompose its channel with $R_{1}$ as well as its channel with $R_{2}$ to create one non-interfering link to $R_{1}$ and another to $R_{2} . T_{2}$ is able to achieve this as well, and each receiver can then decode its message without interference.

In fig. 3, we fix the distance between each transmitter and receiver to be equal to that between $T_{1}$ and $T_{2}$. In this case, the transmitters allocate the same resources to their sharing link as to their transmission links. Fig. 3 indicates that the share-andtransmit scheme always has a lower rate for the same transmit power than the no cooperation scheme, which agrees with our result in section IV.

In fig. 4, the distance between each transmitter and receiver is $5 \times$ that between $T_{1}$ and $T_{2}$. Note that in this case, the sharing link is stronger than the transmission links since it does not suffer any path loss whereas the transmission links do. Fig. 4 shows that share-and-transmit scheme outperforms the no cooperation scheme. As expected, when the sharing link is stronger, cooperation between transmit nodes results in performance improvement over the no cooperation scheme. Note that while our simulations are for the interference channel, similar results have been obtained for the MAC in [13].

\section{CONCLUSIONS}

We investigate the available DoF for MIMO interference channel. The distributed nature of the antennas significantly limits DoF. For an interference channel with a total of $N$ transmit antennas and a total of $N$ receive antennas, the available \# of DoF can vary from $N$ to 1 based on how the antennas are distributed among the two transmitters and receivers. Through an example of a share-and-transmit scheme, we show how the gains of transmitter cooperation are entirely offset by the cost of enabling that cooperation so that the available DoF are not increased.

\section{REFERENCES}

[1] G. J. Foschini and M. J. Gans, "On limits of wireless communications in a fading environment when using multiple antennas," Wireless Personal Commun. : Kluwer Academic Press, no. 6, pp. 311-335, 1998.

[2] E. Telatar, "Capacity of multi-antenna Gaussian channels," European Trans. on Telecomm. ETT, vol. 10, pp. 585-596, November 1999.

[3] L. Zheng and D. N. Tse, "Packing spheres in the Grassmann manifold: A geometric approach to the non-coherent multi-antenna channel,", IEEE Trans. Inform. Theory, vol. 48, pp. 359-383, Feb 2002.

[4] S. Jafar, "Isotropic fading vector broadcast channels: the scalar upperbound and loss in degrees of freedom," To appear in the IEEE Trans. Inform. Theory. See http://newport.eecs.uci.edu/ syed/.

[5] A. Lapidoth, "On the high-SNR capacity of non-coherent networks," Submitted to IEEE Trans. Inform. Theory. See http://arxiv.org/abs/cs.IT/0411098.

[6] S. Jafar, "Degrees of freedom in distributed MIMO communications," IEEE Communication Theory Workshop, 2005.

[7] A. Host-Madsen and Z. Yang, "Interference and cooperation in multisource wireless networks," in IEEE Communication Theory Workshop, June 2005.

[8] A. B. Carliel, "Outer bounds on the capacity of Gaussian interference channels," IEEE Trans. Inform. Theory, vol. 29, pp. 602-606, July 1983.

[9] G. Kramer, "Outer bounds on the capacity of Gaussian interference channels," IEEE Trans. Inform. Theory, vol. 50, pp. 581-586, Mar. 2004.

[10] S. Vishwanath and S. Jafar, "On the capacity of vector Gaussian interference channels," in Proceedings of IEEE Information Theory Workshop, Oct. 2004.

[11] R. Ahlswede, "The capacity region of a channel with two senders and two receivers," in Ann. Prob., pp. 805-814, Oct. 1974.

[12] A. B. Carliel, "A case where interference does not reduce capacity," IEEE Trans. Inform. Theory, vol. 21, pp. 569-570, Sep. 1975.

[13] S. Cui, A. Goldsmith, and A.Bahai, "Energy efficiency of MIMO and cooperative MIMO in sensor networks," IEEE Journal on Selected Areas in Communications, vol. 22, August 2004. 